\title{
Study on Comprehensive Optimization Method in the Performance of a New Type of High-speed USV and Its Analysis
}

\author{
Zifan Wei ${ }^{1}$, Songlin Yang ${ }^{2, *}$, Shengping Jing ${ }^{2}$ and Man Liu ${ }^{2}$ \\ ${ }^{1}$ Jiangsu Modern Shipbuilding Technology Co, Ltd, Zhenjiang, Jiangsu, China \\ ${ }^{2}$ Jiangsu University of Science and Technology, Zhenjiang, Jiangsu, China \\ ${ }^{*}$ Corresponding author
}

\begin{abstract}
Taking the comprehensive performance of a new type of high-speed unmanned surface vehicle (USV) including its rapidity, maneuverability, seakeeping and overturning resistance into consideration, this paper establishes a mathematical model of the performance comprehensive optimization of high-speed USV. And according to the mathematical model and optimization algorithm, comprehensive optimization software based on a variety of optimization algorithms was designed and compiled to analyze the relevant calculation. The results indicates that the optimization effect of the growth mechanism is more superior to the roulette mechanism; genetic algorithm of growth mechanism and particle swarm optimization algorithm would have the best results in the optimization of the 5000th generation, and it can get the rapidity optimization system had greater influence on the total optimization system; and what's more, the optimal values of hull length and other key design variables and partial parameters of different algorithms are obtained.
\end{abstract}

Keywords-high-speed USV; comprehensive optimization; genetic algorithm; particle swarm optimization algorithm; key variables

\section{INTRODUCTION}

USV is a surface ship that can realize autonomous navigation through intelligent control ${ }^{[1]}$. As a kind of surface carrier, it has the advantages of small volume, high speed, intelligence and so on. Some equipments such as radar, sonar, camera, and some small arms can be installed in the USV and these are mainly used to complete the collection and dissemination of information, monitoring the marine situation, combat and other tasks. In addition, with the development of unmanned combat platform in all fields, the USV will be an important tool for the marine combat and exploration of the ocean $^{[2-4]}$

Studying the rapidity performance, seakeeping performance, maneuverability performance and overturning resistance performance of USV is very important for the boat type design and analysis of fluid dynamics performance. And the optimization design of the comprehensive performance for USV has an irreplaceable role in the design and research of USV. Ship design is a complicated process, which needs to be coordinated in a variety of disciplines ${ }^{[5]}$. In recent years, with the rapid development of computer technology and theory of various disciplines, in the process of design and subject analysis, you can use a more accurate calculation model. At the same time, the computer aided design in the field of ship design is developing rapidly, and the ship design becomes more reasonable and flexible ${ }^{[6]}$. With the development of computer application software applied in ship design, optimization is an effective method, which is widely used in ship design and analysis.

\section{THE ESTABLISHMENT OF THE OPTIMIZATION MATHEMATICAL Model OF High-SPEEd USV}

Through considering synthetically with the optimization mathematical model of ship rapidity, maneuverability, seakeeping and overturning resistance, this paper constructs the optimization objective function of comprehensive performance for a high speed USV. According to the constraint conditions, the penalty function is designed, and finally the optimization mathematical model of comprehensive performance for a high speed USV is constructed.

\section{A. Design Variable}

There are many factors affecting the rapidity, maneuverability, seakeeping and overturning resistance of high speed USV. Considering all kinds of factors that affect the performance of the hull, the paper selects the following design variables: hull length( $L$ ), hull width( $B$ ), $\operatorname{draft}(T)$, block coefficient $\left(C_{B}\right)$, mid-ship section coefficient $\left(C_{M}\right)$, designed water plane coefficient $\left(C_{W}\right)$, longitudinal position of buoyancy center( ${ }^{L_{C P}}$ ), propeller diameter $\left({ }^{D_{P}}\right)$, disk area ratio( $\left.{ }^{A_{e o}}\right)$, pitch ratio( $\left.P_{D P}\right)$, rotation speed of propeller $(N)$, design speed $\left(V_{S}\right)$, the ratio of ship gravity distance amid ship to hull length $\left(\delta_{G L}\right)$, the ratio of span hydrofoil to hull width $\left(\delta_{L B}\right)$, hydrofoil aspect $\operatorname{ratio}\left(\delta_{0}\right)$, hydrofoil initial angle of $\operatorname{attack}\left(\alpha_{0}\right)$, the ratio of installation position of hydrofoil distance bow to hull length( $\delta_{L L}$ ), hydrofoil relative submergence $\left({ }^{h_{0}}\right)$, front and back width ratio of splash proof 
$\operatorname{strip}\left(\delta_{f}\right)$, the ratio of the vertical position of the center of gravity to molded depth $\left(\delta_{Z D}\right)$, running pitch angle $(\alpha)$, rise angle of $\operatorname{ramp}(\beta)$, the ratio of the length of the top superstructure to bottom length( $\delta_{L 1}$ ), height of top superstructure(H1), the ratio of the length of the bottom superstructure to hull length( $\left.\delta_{L 2}\right)$, height of bottom superstructure $(\mathrm{H} 2)$, the ratio of superstructure width to hull width $\left(\delta_{\mathrm{Ba}}\right)$, the ratio of draft to molded depth(TD). The 28 design variables are represented by a vector $X_{S P}$ as follows:

$$
\begin{aligned}
X_{S P}=\left\{L, B, T, C_{B}, C_{M}, C_{W}, L_{c p}, D_{P}, A_{e o}, P_{D P}, N, V_{s}, \delta_{G L}\right. \\
\\
\left.\delta_{L B}, \delta_{0}, \alpha_{0}, \delta_{L L}, h_{0}, \delta_{f}, \delta_{Z D}, \alpha, \beta, \delta_{L 1}, H_{1}, \delta_{L 2}, H_{2}, \delta_{B a}, T D\right\}
\end{aligned}
$$

\section{B. Objective Function}

In this paper, the high speed USV has four sub objective functions of rapidity, maneuverability, seakeeping and overturning resistance. The total objective function of the optimization mathematical model is composed of four sub objective functions in the form of power exponent product, and combining the penalty function to get the final fitness function.

\section{1) Total optimization objective function}

The index of USV rapidity, maneuverability, seakeeping and overturning resistance are combined, using the way of power exponent product with each index to obtain the total optimization objective function.

The total optimization objective function is as follows:

$$
F(x)=f_{1}(x)^{\alpha_{1}} * f_{2}(x)^{\alpha_{2}} * f_{3}(x)^{\alpha_{3}} * f_{4}(x)^{\alpha_{4}}
$$

Where $f_{1}(x), f_{2}(x), f_{3}(x), f_{4}(x)$ respectively are objective function of rapidity, maneuverability, seakeeping and overturning resistance, $\alpha_{1}, \alpha_{2}, \alpha_{3}, \alpha_{4}$ respectively are weight of four systems, $\alpha_{1}, \alpha_{2}, \alpha_{3}, \alpha_{4}$ are greater than 0 , and $\alpha 1 * \alpha 2 * \alpha 3 * \alpha 4=1$.

\section{2) Optimization fitness function}

The optimization fitness function is the product of the optimization of the total objective function and the penalty function, and the penalty function is the product of the penalty value of equality constraints and inequality constraints.

The fitness function is as follows:

$$
S=F(x) * P(x)
$$

Where $P(x)$ is penalty function, $P(x)=P_{1}(x) * P_{2}(x)$, $P_{1}(x)$ is the penalty value of equality constraints, $P_{2}(x)$ is the penalty value of inequality constraints.

\section{Constraints Conditions}

In this paper, the constraint conditions of the mathematical model contains 5 equality constraints and 6 inequality constraints.

\section{OPTIMUM ALGORITHM}

\section{A. Genetic Algorithm of Growth Mechanism}

The principle of genetic is to produce better and better individuals in accordance with the mechanism of survival of the fittest. The algorithm is based on the size of the individual fitness value, with a certain rule to select a good individual to the next generation, and randomly selected individuals for crossover and mutation operation, and then generate a new population solution set[7-8]. The general genetic algorithm uses roulette mechanism, the main characteristics of the roulette mechanism are the large probability of the large individual's fitness value is chosen to be the next generation. Such mechanisms tend to result in a large number of repeat individuals in the next generation (individuals with large fitness values), so that the population in the process of evolution has lost the diversity. Taking into account the above reasons, the carrier mechanism was used in genetic algorithm, the improvement idea is, when the initial population is generated, the worst parts individuals of the next generation are randomly generated according to the characteristics of the several best individuals in the last generation in the neighborhood. This can not only ensure the evolution of the population towards the best individual, but also ensure that the population preserve the original features of individuals of the last generation. Of course the accomplish of the improved algorithm involves three parameters: according to the characteristics of the number of individuals to produce a new individual, the range of the neighborhood and the number of the next generation of individuals to be eliminated.

\section{B. Algorithm of Particle Swarm Optimization}

The basic idea of particle swarm optimization algorithm is derived from the migration and clustering characteristics of birds foraging. It is similar to other evolutionary algorithms, and also has the concept of "population" and "evolution". Search for the optimal solution of complex space by the behavior of cooperation and competition among individuals. The algorithm has strong global search capability and is easy to be realized. It has been widely used in the fields of science and engineering[9].

Because the trajectory of the particle position update formula is not controllable, this makes the particle cycle beat in the solution space, so the maximum speed is used to limit the speed. Increase the maximum speed is conducive to global search, reduce the maximum speed is conducive to local development. But the maximum speed is too high, the particle motion may cross the optimal solution in the location, leading 
to the algorithm is difficult to converge; on the contrary, the maximum speed is too small, the algorithm may fall into local extreme value[10]. The choice of maximum speed is usually given by experience, and is generally set to the $10-20 \%$ of the problem space[11].

\section{ANALYSIS OF OPTIMIZATION CALCULATIONS AND RESEARCH ON ALGORITHMS}

According to the importance of each kind of performance, the weight of the four performance respectively as: the weight of rapidity objective function is 2 , the weight of maneuverability objective function is 1.6 , the weight of seakeeping objective function is 0.625 , the weight of overturning resistance objective function is 0.5 . The weight of each subsystem are selected as follows: the weight of maneuverability: the weight of dimensionless criterion number is 1.6 , the weight of head turning index is 0.625 , the weight of minimum relative turning diameter is 1 . The weight of seakeeping: the weight of dimensionless damping coefficient is 0.625 , the weight of pitch index is 1.6 , the weight of heave index is 1 . The weight of overturning resistance: the weight of initial stability high of floating on even keel is 1.6, the weight of initial stability high after overthrow is 0.625 .

\section{A. Optimization Calculation of Genetic Algorithm}

\section{1) Genetic algorithm calculation of two mechanisms}

Parameters are set as follows: calculation number was 1000 population size was 200 , the roulette mechanism and the growth mechanism were used to calculated respectively. In the growth mechanism, variable carrier probability of was $0.0001-$ 0.01 , genetic factor was 0.1 , evolutionary weight was 0.5 . The calculation results of the genetic algorithm of the two mechanisms are shown in TABLE. 1.

\section{TABLE I. CALCULATION RESULTS OF GENETIC ALGORITHM FOR} TWO MECHANISMS

\begin{tabular}{|c|c|c|c|c|}
\hline Calculation number & $\mathbf{1}$ & $\mathbf{2}$ & $\mathbf{3}$ & $\mathbf{4}$ \\
\hline Calculating mechanisms & roulette & roulette & growth & growth \\
\hline Fitness value & $2.33 \mathrm{E}-22$ & $3.48 \mathrm{E}-22$ & 119.62 & 116.31 \\
\hline Penalty function value & $4.99 \mathrm{E}-24$ & $4.70 \mathrm{E}-24$ & 1 & 1 \\
\hline
\end{tabular}

The genetic algorithm of the two mechanisms is calculated two times, as shown in TABLE. 1, the calculation results of the two mechanisms are basically stable. The fitness value and penalty function value of growth mechanism is much larger than the roulette mechanism, we can obtains the calculation results of growth mechanism is much better than the roulette mechanism.

2) Genetic algorithm of variable carrier probability growth mechanism: Variable inner carrier probability: 0.00010.01 .

\section{a) Different evolutionary weights}

The calculation number was 1000 , the genetic factor was set to 0.1 , and the evolutionary weight was calculated by the following 6 groups.
TABLE II. CALCULATION RESULTS OF DIFFERENT EVOLUTIONARY WEIGHTS

\begin{tabular}{|c|c|c|c|c|c|c|}
\hline $\begin{array}{c}\text { Evolutionary } \\
\text { weight }\end{array}$ & $\mathbf{0 . 1}$ & $\mathbf{0 . 3}$ & $\mathbf{0 . 5}$ & $\mathbf{0 . 7}$ & $\mathbf{0 . 9}$ & $\mathbf{0 . 9 5}$ \\
\hline Fitness value & $3.00 \mathrm{E}-35$ & $3.55 \mathrm{E}-36$ & 100.06 & 120.09 & 125.77 & 121.41 \\
\hline $\begin{array}{c}\text { Penalty function } \\
\text { value }\end{array}$ & $3.17 \mathrm{E}-37$ & $2.57 \mathrm{E}-38$ & 1 & 1 & 1 & 1 \\
\hline
\end{tabular}

As shown in TABLE. 2, with the change of evolutionary weight, there is an optimal evolutionary weight. For the mathematical model of this paper, the evolutionary weight is 0.9 , which has better calculation results , and the larger evolutionary weights are better.

\section{b) Different genetic factors}

The calculation number was 1000 , the evolutionary weight was set to 0.9 , and the genetic factor was calculated by the following 7 groups.

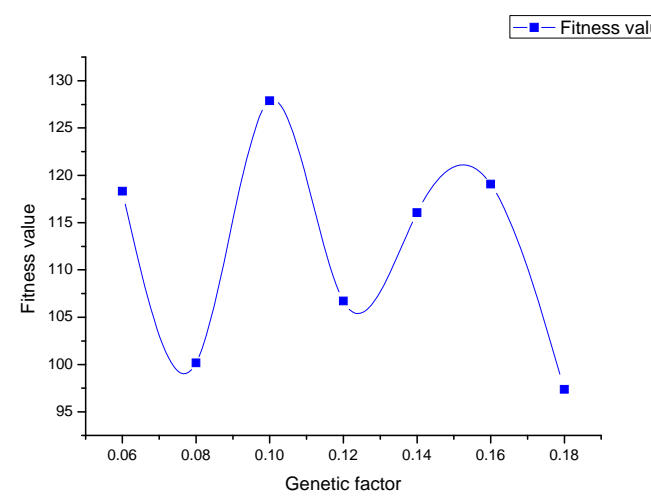

\section{FIGURE I. CHANGE CURVE OF FITNESS VALUE OF DIFFERENT GENETIC FACTORS}

From Figure 1, with the change of genetic factors, the fitness value is also changing, there is an optimal genetic factor. For the mathematical model of this paper, the best genetic factor is 0.1 .

\section{c) Different calculation number}

The evolutionary weight was set to 0.9 , the genetic factor was set to 0.1 . The number of calculations to select a total of 7 sets of 1000, 2000, 3000, 4000, 5000, 6000, 7000, and the results shown in Figure 2.

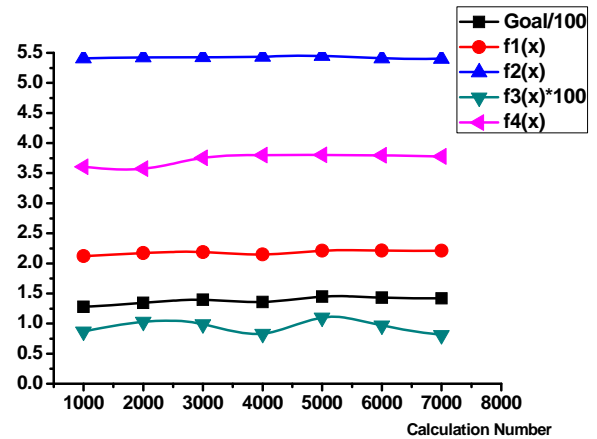

FIGURE II. CHANGE CURVE OF OBJECTIVE FUNCTION VALUE OF DIFFERENT CALCULATION NUMBER 
In Figure 2, Goal is the fitness value, $\mathrm{fl}(\mathrm{x})$ is the rapidity objective function value, $\mathrm{f} 2(\mathrm{x})$ is the maneuverability objective function value, $\mathrm{f} 3(\mathrm{x})$ is the seakeeping objective function value, $\mathrm{f} 4(\mathrm{x})$ is the overturning resistance objective function value. From the variation curves of different objective function values in Figure 2, the calculated number is about 5000, which has the maximum fitness value. The objective function value is very stable, the change trend of the fitness value is consistent with the rapidity system, and it can be concluded that the rapidity optimization system have a large impact for the whole optimization system.

Optimization Calculation of Particle Swarm Optimization Algorithm

Variable weight: $0.9 \sim 0.4$, where the initial weight is 0.9 , the final weight is 0.4 . interval

Different probability of maximum particle flight speed and

The calculation number was 1000 , the probability of maximum particle flight speed and interval was calculated by the following 6 groups.

TABLE III. CALCULATION RESULTS OF DIFFERENT PROBABILITY OF MAXIMUM PARTICLE FLIGHT SPEED AND INTERVAL

\begin{tabular}{|c|c|c|c|c|c|c|}
\hline $\begin{array}{c}\text { probability of maximum } \\
\text { particle flight speed and } \\
\text { interval }\end{array}$ & $\mathbf{0 . 1}$ & $\mathbf{0 . 1 2}$ & $\mathbf{0 . 1 4}$ & $\mathbf{0 . 1 6}$ & $\mathbf{0 . 1 8}$ & $\mathbf{0 . 2}$ \\
\hline Fitness value & 121.77 & 103.16 & 127.33 & 118.51 & 86.135 & 116.50 \\
\hline Penalty function value & 1 & 1 & 1 & 1 & 1 & 1 \\
\hline
\end{tabular}

The speed of particle flight is limited to the range of maximum flight speed. As shown in TABLE. 3, with the increase of the probability of maximum particle flight speed and interval, the fitness function value is changed. In these changing processes, there is an optimal probability of flight speed and interval, when it is 0.14 , the fitness function value is the highest, and the optimal calculation result is the best.

\section{Different calculation number}

The probability of maximum particle flight speed and interval was set to 0.14 , the calculation number was calculated by the following 6 groups.

TABLE IV. CALCULATION RESULTS OF DIFFERENT CALCULATION NUMBER

\begin{tabular}{|c|c|c|c|c|c|c|}
\hline Calculation number & $\mathbf{1 0 0 0}$ & $\mathbf{2 0 0 0}$ & $\mathbf{3 0 0 0}$ & $\mathbf{4 0 0 0}$ & $\mathbf{5 0 0 0}$ & $\mathbf{6 0 0 0}$ \\
\hline Fitness value & 127.33 & 113.96 & 134.02 & 129.26 & 138.80 & 131.05 \\
\hline Penalty function value & 1 & 1 & 1 & 1 & 1 & 1 \\
\hline
\end{tabular}

From the TABLE. 4, when the calculation number is 5000 , have the maximum fitness value, and all meet the constraints.

\section{B. Analysis of the Influence of Key Variables on Optimization System}

The optimization calculation of multiple key design variables, such as hull length and speed is carried out by genetic algorithm of growth mechanism. The changes of their fitness values are obtained, as shown in the following figure.

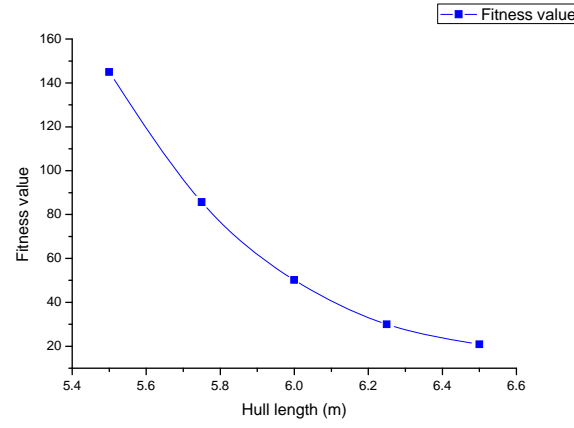

FIGURE III. THE VARIATION OF THE FITNESS VALUE WITH THE HULL LENGTH

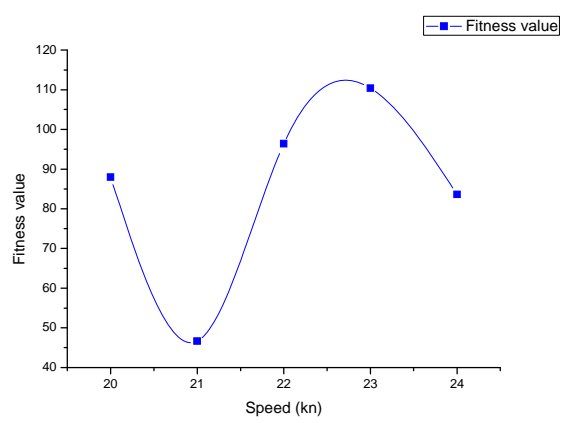

FIGURE IV. THE VARIATION OF THE FITNESS VALUE WITH THE SPEED

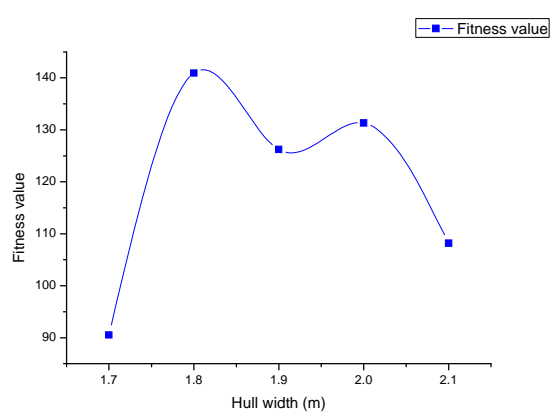

FIGURE V. THE VARIATION OF THE FITNESS VALUE WITH HULL WIDTH

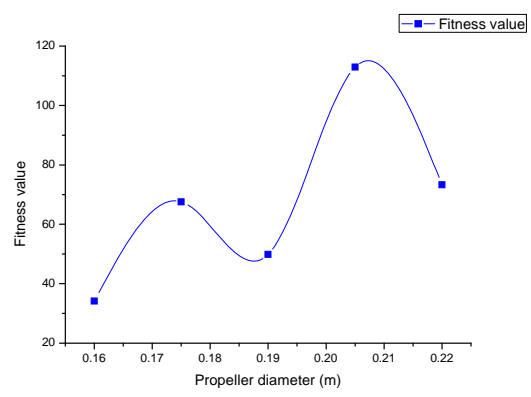

FIGURE VI. THE VARIATION OF THE FITNESS VALUE WITH PROPELLER DIAMETER 
From the above 4 pictures, it can be seen that the fitness value of hull length and other key design variables change significantly, which indicating that they are more sensitive to the total optimization system.

Figure 3 is the variation of the fitness value with hull length, the trend is that with the increase of the hull length, the fitness value has been reduced, note that the smaller the hull length, the better the effect of the optimization, the best hull length is $5.5 \mathrm{~m}$. Figure 4 is the variation of the fitness value with speed, the trend is that with the increase of speed, the fitness value decreases first, then increases, and finally decreases, the best speed is about $22.5 \mathrm{kn}$. Figure 5 is the variation of the fitness value with hull width, the trend is that with the increase of hull width, fitness value increases first, then decreases, then increases, and finally decreases, the best hull width is about $1.8 \mathrm{~m}$. Figure 6 is the variation of the fitness value with propeller diameter, the trend is basically same as the variation of the fitness value with hull width, the best propeller diameter is about $0.21 \mathrm{~m}$.

\section{CONCLUSION}

To summarize, this paper selects a new type of high speed USV as research object, then takes into account four major performance, and finally establishes a comprehensive optimization mathematical model. Through the usage of genetic algorithm, particle swarm optimization algorithm and complex algorithm, combined with external hierarchical and parallel strategy, optimization algorithm was designed, and then comprehensive optimization software was compiled to analyze the relevant calculation. Finally, this paper has got some conclusions as follows:

A. In the genetic algorithm, the result of the growth mechanism is much better than the roulette mechanism.

B. For the mathematical model of this paper, in the genetic algorithm of growth mechanism, the evolutionary weight is 0.9 , which has better calculation results. And the best genetic factor is 0.1 .

C. For the mathematical model of this paper, the calculation results of particle swarm optimization algorithm is best when the probability of maximum particle flight speed and interval is 0.14 .

D. Genetic algorithm of growth mechanism and particle swarm optimization algorithm would have the best results in the optimization of the 5000th generation, and it can get the rapidity optimization system had greater influence on the total optimization system.

E. Several key design variables, such as hull length, are sensitive to the total optimization system. Some conclusions are as follows: the best hull length is $5.5 \mathrm{~m}$, the best speed is about $22.5 \mathrm{kn}$, the best hull width is about $1.8 \mathrm{~m}$, and the best propeller diameter is about $0.21 \mathrm{~m}$.

\section{ACKNOWLEDGMENT}

The project background of this article is project of National Natural Science Foundation of China, Grant number is 51379094.

\section{REFERENCES}

[1] S. Suresh and N. Kannanb. Direct adaptive neural flight control system for an unstable unmanned aircraft [J]. Applied Soft Computing 8 (2008)937-948.

[2] Kuang Xiaomei. Research on the concept design of USV [D]. Harbin Engineering University, 2007.

[3] Li JiaLiang. Development and Application of USV [J]. Fire Control \& Command Control, 2012,06:203-207.

[4] Wan jiexi. Status and Development Trends of Foreign Military Unmanned Surface Boats [J]. National Defense Science \& Technology, 2014,05:91-96.

[5] Apostolos Papanikolaou. Holistic ship design optimization[J]. Computer-Aided Design 42 (2010) 10281044

[6] Horst Nowacki. Five decades of Computer-Aided Ship Design [J]. Computer-Aided Design 42 (2010) 956969.

[7] Wu QiZhong. Operations research and optimization methods [M]. Beijing: Mechanical Industry Press, 2003.

[8] Cao Daoyou. Research and applications based on improved genetic algorithm[D]. Anhui University,2010.

[9] Liu Yi. Improvements and applications of particle swarm optimization algorithm [D]. Xidian University, 2013.

[10] Y. del Valle, G. Venayagamoorthy, S. Mohagheghi, et al. Particle Swarm Optimization: Basic Concepts, Variants and Applications in Power Systems [J]. IEEE Transactions on Evolutionary Computation, vol.12, no.2, pp.171-195, 2008.

[11] Yang Weixin, Zhang Xiaosen. Review of particle swarm optimization algorithm [J]. Gansu Science and Technology, 2012,05:88-92+73. 